\title{
Endogenous glucose production is inhibited by the adipose-derived protein Acrp30
}

\author{
Terry P. Combs, ${ }^{1}$ Anders H. Berg, ${ }^{1}$ Silvana Obici, ${ }^{2,3}$ Philipp E. Scherer, ${ }^{1,3}$ \\ and Luciano Rossetti2,3,4 \\ ${ }^{1}$ Department of Cell Biology, \\ ${ }^{2}$ Department of Medicine, \\ ${ }^{3}$ Diabetes Research and Training Center, and \\ ${ }^{4}$ Department of Molecular Pharmacology, Albert Einstein College of Medicine, Bronx, New York, USA
}

Address correspondence to: Luciano Rossetti, Department of Pharmacology and Diabetes Research and Training Center, Albert Einstein College of Medicine, 1300 Morris Park Avenue, Bronx, New York 10461, USA.

Phone: (718) 430-4118; Fax: (718) 430-8557; E-mail: rossetti@aecom.yu.edu.

T.P. Combs and A.H. Berg contributed equally to this work.

Received for publication August 31, 2001, and accepted in revised form October 15, 2001.

Intraperitoneal injection of purified recombinant Acrp30 lowers glucose levels in mice. To gain insight into the mechanism(s) of this hypoglycemic effect, purified recombinant Acrp30 was infused in conscious mice during a pancreatic euglycemic clamp. In the presence of physiological hyperinsulinemia, this treatment increased circulating Acrp30 levels by approximately twofold and stimulated glucose metabolism. The effect of Acrp30 on in vivo insulin action was completely accounted for by a $65 \%$ reduction in the rate of glucose production. Similarly, glucose flux through glucose-6phosphatase (G6Pase) decreased with Acrp30, whereas the activity of the direct pathway of glucose6-phosphate biosynthesis, an index of hepatic glucose phosphorylation, increased significantly. Acrp30 did not affect the rates of glucose uptake, glycolysis, or glycogen synthesis. These results indicate that an acute increase in circulating Acrp30 levels lowers hepatic glucose production without affecting peripheral glucose uptake. Hepatic expression of the gluconeogenic enzymes phosphoenolpyruvate carboxykinase and G6Pase mRNAs was reduced by more than $50 \%$ following Acrp30 infusion compared with vehicle infusion. Thus, a moderate rise in circulating levels of the adiposederived protein Acrp30 inhibits both the expression of hepatic gluconeogenic enzymes and the rate of endogenous glucose production.

J. Clin. Invest. 108:1875-1881 (2001). DOI:10.1172/JCI200114120.

\section{Introduction}

A close relationship exists between changes in fat mass and the regulation of insulin action $(1,2)$. Insulin resistance and hyperinsulinemia are present in obese as well as lipodystrophic individuals, suggesting that altered adipocyte function may play a role in the regulation of insulin action (1-4). Furthermore, insulinsensitizing drugs that target transcriptional regulation of adipocytes also link insulin responsiveness to adipocyte function $(4,5)$. It is quite likely that marked alterations in lipid fluxes contribute to the pathophysiology of insulin resistance in these syndromes (6-10). However, plasma proteins secreted by adipose tissue may also influence insulin action in other organs.

Speculation about the potential role of Acrp30 (also known as AdipoQ, adiponectin, apM1, and GBP28) in energy balance is based on its discovery in plasma and its exclusive expression in adipose tissue (11). Indeed, circulating levels of Acrp30 are elevated during chronic caloric restriction in both humans and mice $(12,13)$. In contrast, low levels of Acrp30 in human plasma correlate with high fasting insulin, glucose, and triglycerides as well as higher degrees of obesity $(12,14-16)$.
Acrp30 mRNA levels in adipose tissue from obese humans and ob/ob mice are also reduced (17). Furthermore, a diabetes susceptibility locus has been mapped to chromosome $3 \mathrm{q} 27$, the location of the human Acrp30 gene (18).

Correlative evidence supporting an interaction between circulating Acrp30 and insulin sensitivity has recently been reported. Longitudinal studies in rhesus monkeys revealed that plasma Acrp30 levels declined at an early phase of obesity, and continued to decrease after the development of type 2 diabetes (19). Interestingly, in vivo insulin action was most impaired in nondiabetic obese monkeys with the lowest levels of Acrp30. Furthermore, in isolated human populations, plasma levels of Acrp30 were inversely related to fasting insulin levels and insulin resistance (20).

Several laboratories recently examined the effects of Acrp30 on glucose and lipid metabolism. Berg et al. reported that a two- to fivefold elevation in circulating Acrp30 levels transiently reduces plasma glucose levels in wild-type and diabetic mice (13). The glucose lowering effect of Acrp30 did not coincide with a rise in insulin; however, subphysiologic amounts of insulin 
were required for Acrp30 to suppress glucose production in primary hepatocytes. The glucose lowering effect in vivo was also observed with a proteolytic fragment of Acrp30 by Fruebis et al. (21). Chronic administration of Acrp30 or its proteolytic fragment decreased body adiposity and improved glucose tolerance in high-fat-fed as well as lipodystrophic models $(16,21)$. Finally, Acrp30 may be a target of a class of drugs that enhances insulin sensitivity, since treatment of $\mathrm{db} / \mathrm{db}$ mice with agonists of PPAR- $\gamma$ receptors increased circulating Acrp30 levels $(13,16)$.

In the present study, we used the pancreatic insulin clamp technique in conscious mice to determine whether the acute increase in Acrp30 lowers circulating glucose levels by increasing glucose uptake, decreasing glucose production, or both. The results indicate that short-term intravenous infusion of Acrp30 in the presence of modest hyperinsulinemia inhibits glucose production without affecting glucose uptake, glycolysis, or glycogen synthesis. Thus, suppression of hepatic glucose production is the main mechanism by which Acrp30 acutely lowers plasma glucose concentrations. Furthermore, in vivo measurements of hepatic glucose fluxes and Northern blot analyses point to the gluconeogenic enzymes phosphoenolpyruvate carboxykinase (PEPCK) and glucose-6-phosphatase (G6Pase) as potential molecular targets of Acrp30 in the liver.

\section{Methods}

Animals. Adult male FVB mice (30-35 g) were anesthetized intraperitoneally with chloral hydrate (400 $\mathrm{mg} / \mathrm{kg}$ body $\mathrm{wt}$ ) and catheterized through the right internal jugular vein as previously described (22-24). The venous catheter was used for infusion at the same time that blood samples were collected from the tail vein. Each animal was monitored for food intake and weight gain for 4-5 days after surgery, to ensure complete recovery.

Recombinant Acrp30. Acrp30 was isolated and purified from 293-T cells stably expressing Acrp30, as previously described (13).

Measurement of Acrp30 protein levels in plasma. Plasma Acrp30 was measured by semiquantitative Western blotting as described (13). This analysis was possible because Acrp30 protein abundance in both basal and clamp plasma samples was within the linear portion of the standard curve. Blots were analyzed with a PhosphorImager and quantitated with ImageQuant software (both from Molecular Dynamics Inc., Sunnyvale, California, USA).

Pancreatic englycemic clamp studies. Euglycemic clamping was performed in conscious, unrestrained, catheterized mice as previously described (22-24). Food was removed for 5 hours before the beginning of in vivo studies. Mice received a constant infusion of Acrp30 (20 ng/g/body wt $/ \mathrm{min})$ or vehicle at $10 \mu \mathrm{l} / \mathrm{min}$ for 10 minutes, after which the rate was reduced to 1 $\mu \mathrm{l} / \mathrm{min}$ for the remainder of the experiment. The total dose of Acrp30 was approximately $4 \mu \mathrm{g} / \mathrm{g}$ body wt. Glucagon was not infused into either group during the clamp procedure. A solution of glucose (10\%) was infused at a variable rate as required to maintain euglycemia $(6 \mathrm{mM})$. Mice received a constant infusion of HPLC-purified $\left[3-{ }^{3} \mathrm{H}\right]$ glucose $(0.1 \mathrm{mCi} / \mathrm{min}$; NEN Life Science Products, Boston, Massachusetts, USA), insulin $(5 \mathrm{mU} / \mathrm{kg}$ body $\mathrm{wt} / \mathrm{min})$, and somatostatin (5 $\mu \mathrm{g} / \mathrm{kg}$ body wt $/ \mathrm{min}$ ). Thereafter, plasma samples were collected to determine glucose levels (at time $=10,20$, $30,40,50,60,70,80$, and 90 minutes) and the specific activities of $\left[3-{ }^{3} \mathrm{H}\right]$ glucose and tritiated water (at time $=40,50,60,70,80$, and 90 minutes). Consecutive samples were pooled for the assessment of the plasma insulin and Acrp30 levels. Steady-state conditions for both plasma glucose concentration and specific activity were achieved by 40 minutes in these studies. At the end of the in vivo studies, mice were anesthetized (intravenous pentobarbital, $60 \mathrm{mg} / \mathrm{kg}$ body wt), the abdomen was quickly opened, portal blood was collected, and each liver was freeze-clamped in situ with aluminum tongs that were cooled in liquid nitrogen. The time between the injection of anesthesia and the freeze-clamping of tissue samples was less than $60 \mathrm{sec}-$ onds. Tissue samples were stored at $-80^{\circ} \mathrm{C}$ for further analysis. The euglycemic clamp protocol was approved by the Institutional Animal Care and Use Committee of the Albert Einstein College of Medicine.

Analytical procedures. Plasma glucose was measured by the glucose oxidase method (Glucose Analyzer II; Beckman Instruments Inc., Fullerton, California, USA). The rates of glycolysis were estimated as previously described (22). Because tritium on the C-3 position of glucose is lost to water during glycolysis, it is assumed that plasma tritium is present in either tritiated water or $\left[3-{ }^{3} \mathrm{H}\right]$ glucose. Radioactivity of $\left[3-{ }^{3} \mathrm{H}\right]$ glucose in plasma was measured from supernatants of $\mathrm{Ba}(\mathrm{OH})_{2}$ and from $\mathrm{ZnSO}_{4}$ precipitates, after each was evaporated to dryness for the removal of tritiated water. The specific activity of tritiated water in plasma was determined from the total counts of the protein-free supernatant before and after evaporation to dryness (25).

Under steady-state conditions for plasma glucose concentration, the rate of glucose uptake equals the rate of glucose appearance $\left(R_{\mathrm{a}}\right) \cdot R_{\mathrm{a}}$ was determined by dividing the infusion rate for $\left[3-{ }^{3} \mathrm{H}\right]$ glucose (disintegrations per minute) by the specific activity of plasma $\left[3-{ }^{3} \mathrm{H}\right]$ glucose (disintegrations per minute per mg glucose) under steady-state conditions. The rate of glucose production was therefore obtained from the difference between $R_{\mathrm{a}}$ and the rate of glucose infusion. The rate of glycogen synthesis was estimated from the difference between $R_{\mathrm{a}}$ and the rate of glycolysis. The percentage of the hepatic glucose-6-phosphate pool directly derived from plasma glucose (direct pathway) was calculated as the ratio of liver $\left[3-{ }^{3} \mathrm{H}\right] \mathrm{UDP}$-glucose specific activity to plasma $\left[3-{ }^{3} \mathrm{H}\right]$ glucose specific activity $(26)$.

RNA preparation and Northern blot analysis. Total RNA from liver was extracted using TRI Reagent (Molecular Research Center Inc., Cincinnati, Ohio, USA) according to the manufacturer's recommendations. Northern 
blot analysis was performed as described previously. Total RNA $(30 \mu \mathrm{g})$ was loaded in each lane. The agarose gel was stained with ethidium bromide to ensure equal loading of the total RNA samples. After transfer of RNA onto a nylon filter by capillary action, the filter was stained with methylene blue to ensure that an equal amount of RNA had transferred. Subclones of murine PEPCK and G6Pase cDNA were isolated by RT PCR using the following sense and antisense primers: 5' PEPCK: ACCCCGAAGGCAAGAAGAAATAC; 3' PEPCK: GCGTCCGAACATCCACTCCAGCAC; 5' G6Pase: GCATCTGTCAGTCTTATCC; and 3' G6Pase: GTACAGTGGAGACTATCTG. Radiolabeled probes were prepared by the random primer labeling method (Life Technologies Inc., Gaithersburg, Maryland, USA) using [ $\left.{ }^{32} \mathrm{P}\right] \mathrm{dCTP}$ (Amersham Pharmacia Biotech, Piscataway, New Jersey, USA). After high stringency hybridization and washing, the membranes were exposed to a Storage Phosphor Screen (Molecular Dynamics Inc.). Quantitative densitometry was achieved by scanning laser using a PhosphorImager (Molecular Dynamics Inc.).

Statistical analysis. Data are presented as mean \pm SE. Statistical analysis was performed using an unpaired, nonparametric Student $t$ test. Differences were considered statistically significant at $P<0.05$.

\section{Results}

General characteristics of the experimental animals. To delineate the effect of increased circulating Acrp30 on peripheral and hepatic insulin action, 12 conscious mice were divided into two groups. One group received a primed-constant infusion of recombinant Acrp30, and the other received a similar infusion of vehicle. There were no differences in the mean body weights between the two groups of mice. Following a fast of approximately 6 hours (postabsorptive state), the plasma insulin and glucose concentrations were similar in the two groups (Table 1).
Pancreatic euglycemic clamp studies. In order to assess the metabolic effects of insulin in vivo, a similar modest increase in the plasma insulin concentrations was generated, and the plasma glucose concentrations were maintained at approximately $6 \mathrm{mM}$ by a variable glucose infusion. Thus, the effects of Acrp30 and vehicle were compared in the presence of similar steady-state insulinemia and normoglycemia (Table 1) in conscious mice. Steady-state conditions for plasma glucose specific activity were achieved within 40 minutes during clamp periods (Table 2). Similarly, the rate of accumulation of tritiated water in the plasma compartment, which reflects its generation from $\left[3-{ }^{3} \mathrm{H}\right]$ glucose and is used in the calculation of the rate of glycolysis, was linear during the last 50 minutes of the clamp study. The intravenous infusion of recombinant Acrp30 promptly raised circulating Acrp30 concentrations by approximately twofold (Figure 1a).

Effect of hyperinsulinemia on glucose disposal and on the partitioning of glucose fluxes. The effect of a similar increase in circulating insulin concentrations on the rates of glucose infusion and tissue glucose uptake is displayed in Figure 1, b and c. All measurements were performed during the final 50 minutes of the clamp study, a time when steady-state conditions were achieved for plasma glucose and insulin concentrations, glucose specific activity, and rates of glucose infusion. The rates of exogenous glucose infusion (Figure $1 b)$ required to maintain the target plasma glucose concentration during the hyperinsulinemic clamp study were increased by $73 \%$ in Acrp30-infused mice compared with vehicle-infused mice. However, infusion with recombinant Acrp30 did not significantly alter the rates of glucose uptake $(27.8 \pm 3.0$ vs. $26.0 \pm 2.1 \mathrm{mg} / \mathrm{kg}$ body wt $/ \mathrm{min})$, glycolysis $(23.1 \pm 1.8$ vs. $22.8 \pm 1.9 \mathrm{mg} / \mathrm{kg}$ body wt $/ \mathrm{min}$ ), or glycogen synthesis (4.6 \pm 1.6 vs. $3.3 \pm 1.1 \mathrm{mg} / \mathrm{kg}$ body wt $/ \mathrm{min}$ ) in Acrp30 and vehicle treatments (respective values; Figure 1c). a

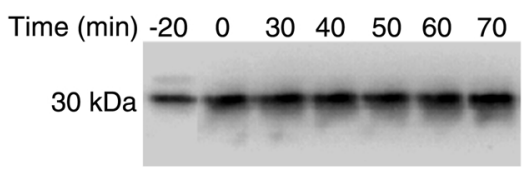

\section{b}

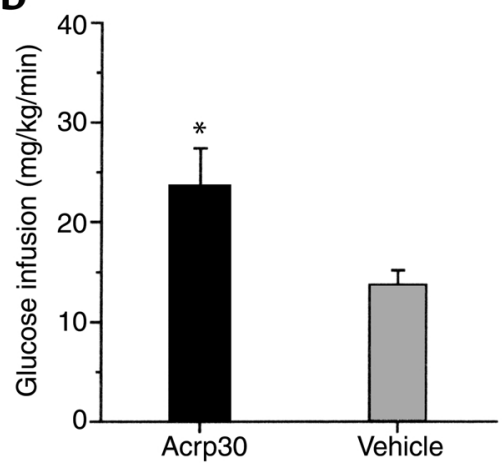

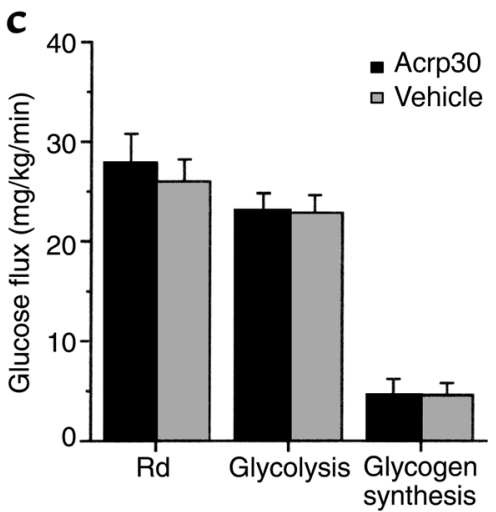

\section{Figure 1}

(a) Time course of plasma Acrp30 during euglycemic clamps. Representative Western blot analysis of plasma from an Acrp30-infused mouse. An affinity-purified, ${ }^{125}$-labeled rabbit anti-mouse Acrp30 antibody was used. A single band was recognized at 30 kDa that was increased by approximately twofold $(P<0.001)$ from baseline (time $=0)$. (b) Effect of Acrp30 on the rate of glucose infusion. (c) Effect of Acrp30 on the rates of glucose disappearance $(\mathrm{Rd})$, glycolysis, and glycogen synthesis. All measurements were obtained while plasma glucose concentration was maintained at approximately $6 \mathrm{mM}$ under steady-state conditions. ${ }^{*} P<0.05$ vs. vehicle. 
Table 1

General characteristics of mice receiving Acrp30 or vehicle before and during euglycemic clamp studies

\begin{tabular}{lcc}
${ } }$ & Vehicle & Acrp30 \\
$n$ & 6 & 6 \\
Bodywt $(\mathrm{g})$ & $31.9 \pm 1.2$ & $32.0 \pm 1.8$ \\
Basal & & \\
Glucose $(\mathrm{mM})$ & $5.8 \pm 0.5$ & $5.6 \pm 0.4$ \\
Insulin $(\mu \mathrm{U} / \mathrm{ml})$ & $23 \pm 3$ & $22 \pm 5$ \\
Clamp & & \\
Glucose $(\mathrm{mM})$ & $6.0 \pm 0.3$ & $5.9 \pm 0.3$ \\
Insulin $(\mu \mathrm{U} / \mathrm{ml})$ & $32 \pm 5$ & $31 \pm 4$ \\
\hline
\end{tabular}

Mice were fasted for 6 hours prior to plasma sampling. Biochemical parameters represent the mean $\pm \mathrm{SE}$.

Effect of recombinant Acrp30 infusion on hepatic glucose fluxes. Figure 2 depicts the rate of endogenous glucose production during the pancreatic insulin clamp procedure. In the presence of similar plasma insulin concentrations, glucose production was suppressed by Acrp30. In fact, this marked improvement in the inhibition of glucose production completely accounted for the increased rate of glucose infusion during the clamp studies. Next, we examined the relative contribution of plasma glucose to the hepatic glucose-6-phosphate pool to determine the effect of Acrp30 on liver glucose fluxes. Table 2 displays the $\left[3-{ }^{3} \mathrm{H}\right]$ UDP-glucose and $\left[3-{ }^{3} \mathrm{H}\right]$ glucose specific activities used to calculate the contribution of plasma glucose (Direct, Table 2) to the hepatic glucose-6-phosphate pool. The ratio of the specific activity of $3-{ }^{3} \mathrm{H}$-labeled hepatic UDP-glucose to that of portal vein plasma glucose provided an estimate of the contribution of hepatic glucose phosphorylation (direct pathway). As shown in Table 2, the contribution of the direct pathway to the hepatic UDP-hexose pool measured at the end of the clamp studies was increased by $55 \%$ in mice receiving Acrp30 compared with vehicle-infused mice. Data obtained on UDPgalactose specific activity confirmed the results obtained with UDP-glucose. These data allowed us to estimate the in vivo fluxes through G6Pase and the rates of glucose cycling in the two groups of mice. As shown in Figure 2, the flux through G6Pase was decreased significantly by Acrp30, in parallel to the effect on glucose production. However, despite this marked decrease in overall glucose output, the rate of glucose cycling was unchanged in Acrp30-infused mice compared with that in vehicleinfused mice. Thus, short-term infusion of the adiposederived protein Acrp30 leads to marked suppression of in vivo G6Pase flux, whereas the direct hepatic phosphorylation of glucose appears to be enhanced.

Effect of recombinant Acrp30 infusion on hepatic PEPCK and G6Pase mRNA. Based on the above flux data, we next explored two potential molecular targets of Acrp30 action in the liver. PEPCK and G6Pase are important determinants of hepatic glucose fluxes, and their regulation by insulin involves transcriptional events. Therefore, we assessed the effect of Acrp30 infusion on PEPCK and G6Pase gene expression in liver harvested at the completion of the clamp studies. Northern blot analyses revealed a marked decrease in G6Pase ( $33 \pm 8$ and $77 \pm 10$ arbitrary units (AU), Acrp30 infusion and vehicle infusion, respectively; $P<0.05)$ and PEPCK (45 \pm 12 and $87 \pm 15 \mathrm{AU}$, Acrp30 infusion and vehicle infusion, respectively; $P<0.05)$ mRNA levels (Figure 2). Thus, the adipose-derived protein Acrp30 decreased the hepatic expression of the gluconeogenic enzymes PEPCK and G6Pase by $48 \%$ and $57 \%$, respectively.

\section{Discussion}

Across species, insulin resistance is strongly linked to obesity $(1,2)$. In the short-term, overfeeding rapidly leads to impaired hepatic and then peripheral insulin action in humans (27) and animals (28). The molecular basis for these associations remains elusive. Increased flux of free fatty acids has been shown to rapidly induce hepatic and peripheral insulin resistance, and thus may play a significant role in these forms of insulin resistance $(6-10,29)$. However, it is also becoming increasingly evident that adipose tissue is not just a site of energy storage, but is also an active endocrine

Table 2

Specific activities of plasma glucose and hepatic substrates

\begin{tabular}{|c|c|c|c|c|c|c|}
\hline Time (min) & 40 & 50 & $\begin{array}{c}60 \\
\mathrm{dpm} / \mathrm{nmol}\end{array}$ & 70 & 80 & 90 \\
\hline Vehicle & $48.3 \pm 7.1$ & $47.9 \pm 7.5$ & $47.5 \pm 6.9$ & $47.7 \pm 6.4$ & $45.6 \pm 5.7$ & $46.1 \pm 6.3$ \\
\hline Acrp30 & $48.3 \pm 5.7$ & $48.7 \pm 7.9$ & $49.6 \pm 6.7$ & $49.4 \pm 5.2$ & $44.8 \pm 7.9$ & $45.9 \pm 5.9$ \\
\hline \multirow[t]{3}{*}{ Group } & {$\left[3-{ }^{3} \mathrm{H}\right]$} & {$\left[3-{ }^{3} \mathrm{H}\right]$} & {$\left[3-{ }^{3} \mathrm{H}\right]$} & & \multicolumn{2}{|l|}{ Direct } \\
\hline & Plasma Glc & UDP-Glc & UDP-Gal & & \multirow{2}{*}{\multicolumn{2}{|c|}{ Gal }} \\
\hline & & $\mathrm{dpm} / \mathrm{nmol}$ & & & & \\
\hline Vehicle & $44.6 \pm 4.5$ & $7.1 \pm 1.4$ & $7.7 \pm 1.6$ & & \multicolumn{2}{|c|}{$16.1 \pm 1.3$} \\
\hline Acrp30 & $46.7 \pm 7.9$ & $12.0 \pm 3.1$ & $13.1 \pm 2.8$ & & $24.0 \pm 3.2^{*}$ & $24.8 \pm 2.9^{*}$ \\
\hline
\end{tabular}

The time course of plasma glucose specific activity documents the achievement of steady-state conditions during the clamp procedure. The specific activity of hepatic substrates was used to calculate the "direct pathway" at the end of the $\left[3-{ }^{3} \mathrm{H}\right]$ glucose infusions in vehicle- and Acrp30-infused mice. Glc, glucose; Gal, galactose; Direct, percentage of the hepatic G6Pase pool derived from plasma glucose, calculated as the ratio of the specific activity of liver [3-3 $\mathrm{H}$ ] UDP-Glc or $\left[3-{ }^{3} \mathrm{H}\right]$ UDP-Gal to that of plasma $\left[3-{ }^{3} \mathrm{H}\right] \mathrm{Glc}$. 
organ (2). Among the numerous circulating proteins that are derived at least partly from adipose cells, some have potent effects on energy metabolism, intermediary metabolism, and insulin signaling (30-35). Leptin may act as a positive modulator of insulin (30-33). TNF- $\alpha$ (34)and resistin act as antagonists of insulin action (35). The presence of severe insulin resistance in models of lipodystrophy $(4,32,36-38)$ and the ongoing search into the mode of action of PPAR- $\gamma$ agonists as insulin sensitizers $(5,36,37)$ lend additional support to this "endocrine hypothesis." In fact, lack of adipose tissue appears to result in impaired insulin action due at least in part to the lack of adipose-derived circulating proteins, such as leptin $(4,32,38)$. Furthermore, PPAR- $\gamma$ agonists may regulate the biosynthesis and secretion of adipose-derived proteins, including resistin (30) and Acrp30 (13). In this regard, it is of interest that PPAR- $\gamma$ agonists are partly effective in the treatment of obesity-linked type 2 diabetes in aP2/DTA mice whose adipose tissue was partially eliminated by the fat-specific expression of diphtheria toxin, but are not effective in treatment of A-ZIP/F-1 mice that have a complete lack of white adipose tissue $(36,37)$.

Two recent publications indicate that intraperitoneal injection of either the full-length or the globular domain of Acrp30 acutely lowers blood glucose levels in mice $(13,21)$. This effect was observed within $2-4$ hours of the injection. Importantly, the Acrp30induced decline in plasma glucose levels was not accompanied by a significant decrease in plasma insulin levels. Indeed, plasma insulin concentrations were moderately increased (by $72 \%$ ) in response to globular Acrp30 fragment in one study (21). In the other study, the plasma insulin concentration failed to decrease despite lowering of the plasma glucose concentrations in response to full-length Acrp30 (13); this study also reported an increase in plasma glucagon levels. These reports indicate that circulating Acrp30 could play a role in the regulation of glucose homeostasis. These effects may be due to subtle alterations in glucoregulatory hormones, to decreased rates of glucose production, and/or to increased rates of glucose uptake. Corollary experiments in ex vivo systems suggested an effect of Acrp30 in the regulation of glucose output from isolated hepatocytes (13) and in the regulation of fat oxidation in isolated muscle strips and in a muscle cell line (21).

To gain insight into mechanisms that may be responsible for the blood glucose lowering effect of Acrp30, we performed pancreatic insulin clamps in conscious mice in combination with intravenous infusion of fulllength Acrp30 or vehicle. This experimental design was selected in order to minimize confounding effects of hypoglycemia and its attendant counterregulatory a

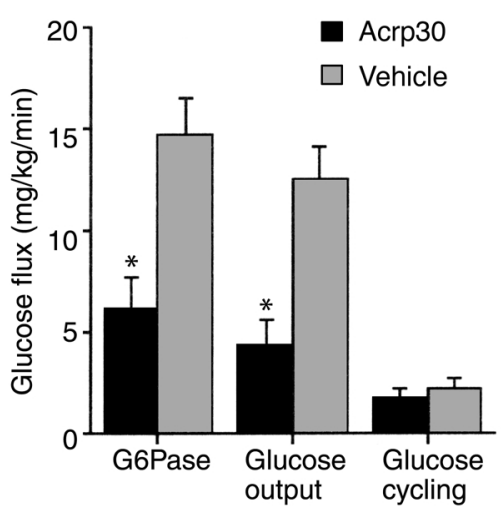

C

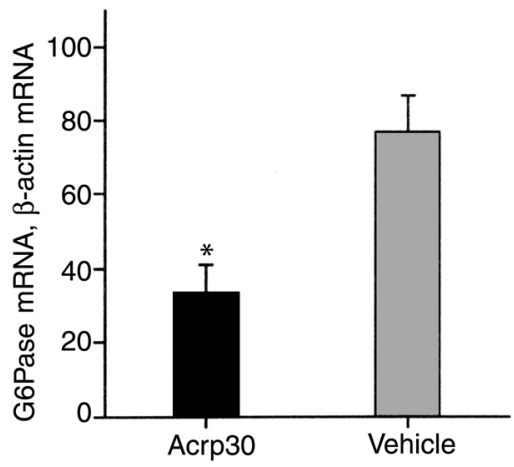

b

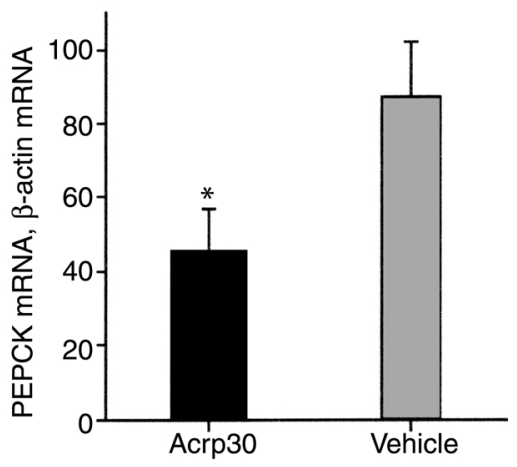

d

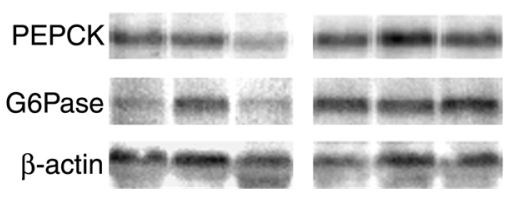

Figure 2

(a) Effect of Acrp30 on the rates of glucose production, G6Pase flux, and glucose cycling. The measurements were obtained while plasma glucose concentration was maintained at approximately $6 \mathrm{mM}$ under steady-state conditions. (b) Effect of Acrp30 on hepatic mRNA expression of PEPCK. (c) Effect of Acrp30 on G6Pase. (d) Representative Northern blots of liver samples from Acrp30-infused (first three lanes) and vehicle-infused (last three lanes) mice. 
responses on metabolic and molecular parameters. Furthermore, the administration of somatostatin served to prevent changes in glucoregulatory hormones such as insulin and glucagon during the clamp procedure. Although the inhibitory effect of somatostatin on plasma glucagon levels may have restrained glucose production, this effect was similar in both groups. Finally, administration of glucose tracer allows one to measure glucose fluxes in vivo. Under these conditions, the intravenous infusion of Acrp30 rapidly reduced the rate of glucose production, but did not alter peripheral glucose fluxes. The present study provides the first in vivo evidence, to our knowledge, that an increase in circulating Acrp30 levels markedly inhibits glucose production in the presence of experimentally controlled and physiological insulin levels. It should be noted that while it is likely that these rapid effects of full-length Acrp30 on hepatic glucose fluxes account for its acute hypoglycemic action, they do not negate additional acute or chronic effects of circulating Acrp30 on glucoregulatory hormones, energy balance, and lipid metabolism $(16,21)$.

The Acrp30 used in this study was identical to the material isolated for the previous study by Berg et al. (13). However, the total amount of Acrp30 infused into each mouse in the present study was only $4 \mu \mathrm{g} / \mathrm{g}$ body $\mathrm{wt}$, whereas a minimum of $28 \mu \mathrm{g} / \mathrm{g}$ body wt was required to lower blood glucose levels in the previous study (13). The detectable onset of Acrp30's metabolic effects also appeared to be more rapid in the present study. There are a few considerations that may help reconcile these findings. First, the route and mode of administration may play a major role. Intravenous administration as a primed constant infusion is likely to maximize the efficacy and minimize the clearance of Acrp30. Of interest, following intraperitoneal bolus injections, the peak of circulating Acrp30 levels occurred 1-4 hours after the bolus, with a prolonged plateau in circulating levels thereafter. This is consistent with slow absorption of the protein from the peritoneal cavity into the plasma compartment. Overall, intravenous delivery may more closely resemble Acrp30 secretion from adipose cells. Second, the measurement of glucose output during pancreatic clamp conditions may be a more sensitive index of the metabolic actions of Acrp30 than its blood glucose lowering effects. In fact, administration of insulin sensitizers to nondiabetic animals generally results in lowering of the plasma insulin concentrations, with minimal changes in the plasma glucose levels. However, high levels of insulin sensitizers can overwhelm the physiological glucoregulatory responses and result in significant lowering of plasma glucose levels. Thus, it is likely that larger and more prolonged elevations in circulating Acrp30 levels may be required to achieve a significant and consistent decline in the plasma glucose concentrations than are needed to suppress glucose output.

In the presence of pancreatic clamp, short-term infusion of recombinant Acrp30 led to a marked suppression of endogenous glucose production in conscious mice. To begin investigating the mechanisms by which Acrp30 decreases the net production of glucose by the liver, we estimated the overall rate of glucose output (in vivo flux through G6Pase) and the relative contribution of hepatic glucose phosphorylation to G6Pase flux. Our assessment of G6Pase flux revealed an approximately $60 \%$ decrease with infusion of Acrp30 compared with vehicle infusion. This decline closely paralleled that seen in net glucose production, suggesting that inhibition of G6Pase by Acrp30 is a likely mode of action. Of interest, whereas the rate of glucose output was markedly decreased in response to Acrp30 infusion, the rate of glucose cycling was not changed. This was due to a marked increase in the contribution of plasma glucose to the hepatic glucose-6-phosphate pool. The latter observation is consistent with Acrp30 preserving the in vivo flux through glucokinase. Finally, we also assessed the effect of Acrp30 on the hepatic expression of PEPCK and G6Pase. Acrp30 markedly decreased levels of G6Pase mRNA and PEPCK mRNA in the liver. While it is likely that the rapid metabolic effects of Acrp30 are largely due to modulation of enzymatic activity, transcriptional regulation of these two key enzymes may also contribute to its molecular mechanism of action.

The discovery of potent metabolic actions by this circulating protein that is exclusively expressed in differentiated adipose cells further supports the notion of crosstalk between energy storage (adipose tissue) and main insulin target organs such as the liver. In this regard, it is intriguing that Acrp30 shares sequence homology with a family of hibernation-regulated proteins, hib 20, 25, and 27, which are differentially expressed in the livers of active and hibernating animals (21). In fact, alterations in energy metabolism during hibernation are tightly linked to dramatic changes in insulin sensitivity and metabolism of lipid and carbohydrate in the liver. Future studies will be needed to delineate whether and how nutrients and changes in energy storage regulate Acrp30 biosynthesis and its circulating levels.

\section{Acknowledgments}

This work was supported by grants from the NIH (R01 DK-45024 and R01 DK-48321 to L. Rossetti, and R01 DK-55758 to P.E. Scherer), the American Diabetes Association (to P.E. Scherer), and by the core laboratories of the Albert Einstein Diabetes Research and Training Center (P60-DK-20541). The work was also supported by the Hormones/Membrane Interactions Training Grant (NIH T32 DK-07513-15 to T.P. Combs) and the Training Program in Cellular \& Molecular Biology \& Genetics (grant T32 GM-07491, to A.H. Berg).

\footnotetext{
1. Boden, G. 1997. Role of fatty acids in the pathogenesis of insulin resistance and NIDDM. Diabetes. 46:3-10.

2. Kahn, B.B., and Flier, J.S. 2000. Obesity and insulin resistance. J. Clin Invest. 106:473-481.

3. Seip, M., and Trygstad, O. 1996. Generalized lipodystrophy, congenital and acquired (lipoatrophy). Acta Paediatr. Suppl. 413:2-28.

4. Reitman, M.L., Arioglu, E., Gavrilova, O., and Taylor, S.I. 2000. Lipoatrophy revisited. Trends Endocrinol. Metab. 11:410-416.
} 
5. Olefsky, J.M., and Saltiel, A.R. 2000. PPAR gamma and the treatment of insulin resistance. Trends Endocrinol. Metab. 11:362-368.

6. Boden, G., Chen, X., Ruiz, J., White, J.V., Rossetti, L. 1994. Mechanisms of fatty acid-induced inhibition of glucose uptake. J. Clin. Invest. 93:2438-2446.

7. Bergman, R.N., et al. 2001. Central role of the adipocyte in the metabolic syndrome. J. Investig. Med. 49:119-126.

8. Sindelar D.K., et al. 1997. The role of fatty acids in mediating the effects of peripheral insulin on hepatic glucose production in the conscious dog. Diabetes. 46:187-196.

9. Kim, J.K., et al. 2001. Tissue-specific overexpression of lipoprotein lipase causes tissue-specific insulin resistance. Proc. Natl. Acad. Sci. USA 98:7522-7527.

10. Dresner A., et al. 1999. Effects of free fatty acids on glucose transport and IRS 1-associated phosphatidylinositol 3-kinase activity.J. Clin. Invest. 103:253-259.

11. Scherer, P.E., Williams, S., Fogliano, M., Baldini, G., and Lodish, H.F. 1995. A novel serum protein similar to C1q, produced exclusively in adipocytes. J. Biol. Chem. 270:26746-26749.

12. Hotta, K., et al. 2000. Plasma concentrations of a novel, adipose-specific protein, adiponectin, in type 2 diabetic patients. Arterioscler. Thromb. Vasc. Biol. 20:1595-1599.

13. Berg, A.H., Combs, T.P., Du, X., Brownlee, M., and Scherer, P.E. 2001. The adipocyte-secreted protein Acrp30 enhances hepatic insulin action. Nat. Med. 7:947-952.

14. Arita, Y., et al. 1999. Paradoxical decrease of an adipose-specific protein, adiponectin, in obesity. Biochem. Biophys. Res. Commun. 257:79-83.

15. Takahashi, M., et al. 2000. Genomic structure and mutations in adiposespecific gene, adiponectin. Int. J. Obes. Relat. Metab. Disord. 24:861-868.

16. Yamauchi, T., et al. 2001. The fat-derived hormone adiponectin reverses insulin resistance associated with both lipoatrophy and obesity. Nat. Med. 7:941-946.

17. Hu, E., Liang, P., and Spiegelman, B.M. 1996. AdipoQ is a novel adiposespecific gene dysregulated in obesity. J. Biol. Chem. 271:10697-10703.

18. Vionnet, N., et al. 2000. Genomewide search for type 2 diabetes-susceptibility genes in French whites: evidence for a novel susceptibility locus for early-onset diabetes on chromosome 3q27-qter and independent replication of a type 2-diabetes locus on chromosome 1q21-q24. Am. J. Hum. Genet. 67:1470-1480.

19. Hotta, K., et al. 2001. Circulating concentrations of the adipocyte protein adiponectin are decreased in parallel with reduced insulin sensitivity during the progression to type 2 diabetes in rhesus monkeys. Diabetes. 50:1126-1133.

20. Weyer, C., et al. 2001. Hypoadiponectinemia in obesity and type 2 diabetes: close association with insulin resistance and hyperinsulinemia. $J$. Clin. Endocrinol. Metab. 86:1930-1935.

21. Fruebis, J., et al. 2001. Proteolytic cleavage product of 30-kDa adipocyte complement-related protein increases fatty acid oxidation in muscle and causes weight loss in mice. Proc. Natl. Acad. Sci. USA. 98:2005-2010.
22. Massillon, D., et al. 1995. Quantitation of hepatic glucose fluxes and pathways of hepatic glycogen synthesis in conscious mice. Am. J. Physiol. 269:E1037-E1043.

23. Rossetti, L., et al. 1997. Peripheral but not hepatic insulin resistance in mice with one disrupted allele of the glucose transporter type 4 (GLUT4) gene. J. Clin. Invest. 100:1831-1839.

24. Rossetti, L., et al. 1997. Abnormal regulation of HGP by hyperglycemia in mice with a disrupted glucokinase allele. Am. J. Physiol. 273:E743-E750.

25. Rossetti, L., and Giaccari, A. 1990. Relative contribution of glycogen synthesis and glycolysis to insulin-mediated glucose uptake. A doseresponse euglycemic clamp study in normal and diabetic rats. J. Clin. Invest. 85:1785-1792.

26. Rossetti, L., et al. 1993. Mechanism by which hyperglycemia inhibits hepatic glucose production in conscious rats. Implications for the pathophysiology of fasting hyperglycemia in diabetes. J. Clin. Invest. 92:1126-1134.

27. Clore, J.N., Helm, S.T., and Blackard, W.G. 1995. Loss of hepatic autoregulation after carbohydrate overfeeding in normal man. J. Clin. Invest. 96:1967-1972.

28. Oakes, N.D., Cooney, G.J., Camilleri, S., Chisholm, D.J., and Kraegen, E.W. 1997. Mechanisms of liver and muscle insulin resistance induced by chronic high-fat feeding. Diabetes. 46:1768-1774.

29. Hawkins, M., et al. 1997. Role of the glucosamine pathway in fat-induced insulin resistance. J. Clin. Invest. 99:2173-2182.

30. Liu, L., et al. 1998. Intracerebroventricular leptin regulates hepatic but not peripheral glucose fluxes. J. Biol. Chem. 273:31160-31167.

31. Sivitz, W.I., Walsh, S.A., Morgan, D.A., Thomas, M.J., and Haynes, W.G. 1997. Effects of leptin on insulin sensitivity in normal rats. Endocrinolo gy. 138:3395-3401.

32. Shimomura, I., Hammer, R.E., Ikemoto, S., Brown, M.S., and Goldstein, J.L. 1999. Leptin reverses insulin resistance and diabetes mellitus in mice with congenital lipodystrophy. Nature. 401:73-76.

33. Kim, Y.B., Uotani, S., Pierroz, D.D., Flier, J.S., and Kahn, B.B. 2000. In vivo administration of leptin activates signal transduction directly in insulin-sensitive tissues: overlapping but distinct pathways from insulin. Endocrinology. 141:2328-2339.

34. Uysal, K.T., Wiesbrock, S.M., Marino, M.W., and Hotamisligil, G.S. 1997. Protection from obesity-induced insulin resistance in mice lacking TNFalpha function. Nature. 389:610-614.

35. Steppan, C.M., et al. 2001. The hormone resistin links obesity to diabetes. Nature. 409:307-312.

36. Burant, C.F., et al. 1997. Troglitazone action is independent of adipose tissue. J. Clin. Invest. 100:2900-2908.

37. Chao, L., et al. 2000. Adipose tissue is required for the antidiabetic, but not for the hypolipidemic, effect of thiazolidinediones. J. Clin. Invest. 106:1221-1228.

38. Gavrilova, O., et al. 2000. Surgical implantation of adipose tissue reverses diabetes in lipoatrophic mice. J. Clin. Invest. 105:271-278. 